\title{
La producción escrita de los estudiantes universitarios de nuevo ingreso
}

Written Work of University Newly Admitted Students

La production écrite des étudiants universitaires de nouvelle admission

A produção escrita dos discentes universitários de recente admissão

Fecha de recepción: 8 DE JULIO DE 2013 / Fecha de aceptación: 6 DE MAYO DE 2015 / Fecha de disponibilidad en línea: 30 DE OCTUBRE DE 2015

Encuentre este artículo en http://magisinvestigacioneducacion.javeriana.edu.co/

Escrito por Manuela Álvarez-Álvarez

UnIVERSIDAD DE DEusto Bilbao, España

manuela.alvarez@deusto.es

María del Mar Boillos-Pereira

UnIVERSIDAD DE DEUSTO

BILbao, España

mmboillos@deusto.es

\begin{abstract}
Resumen
Este artículo de investigación presenta un análisis de las producciones escritas por estudiantes universitarios recién ingresados en un contexto de examen. El objetivo es ofrecer una descripción pormenorizada de la naturaleza lingüísticodiscursiva de estas producciones, e identificar algunos problemas asociados a la composición de este tipo de textos. Para este fin se lleva a cabo un estudio cuantitativo de cuatro niveles de análisis: la estructura, el planteamiento retórico, el nivel lingüístico y el uso de la información. Los desafíos que emergen de tales producciones proporcionan indicios valiosos para la elaboración de propuestas didácticas orientadas a la mejora de la enseñanza de esta competencia.
\end{abstract}

\section{Palabras clave}

Desarrollo de habilidades, formación profesional superior, alfabetización académica

\section{Transferencia a la práctica}

Los resultados de estudio buscan contribuir al avance en la línea de investigación sobre escritura en los medios universitarios. De manera adicional, el trabajo que aquí se presenta tiene como finalidad servir como base para promover mejoras didácticas que favorezcan el tratamiento de las diferentes dificultades que manifiestan los estudiantes universitarios en los distintos estadios de desarrollo de su competencia escritora.

Para citar este artículo / To cite this article / Pour citer cet article / Para citar este artigo Álvarez-Álvarez, M. \& Boillos-Pereira, M. (2015). La producción escrita de los estudiantes universitarios de nuevo ingreso. magis, Revista Internacional de Investigación en Educación, 8(16), 71-90. http://dx.doi.org/10.11144/Javeriana.m8-16.peeu 


\section{Keywords}

Development of skills; higher education teaching development; academic literacy

\section{Mots clés}

Développement d'habilités; formation professionnelle supérieure; alphabétisation académique

\section{Abstract}

This paper presents an analysis of the written work of newly admitted undergraduate students in a context of examination. The aim of the paper is to provide a detailed description of the linguistic-discursive nature of these productions, and identify some problems associated with the composition of such texts. This analysis is made through a quantitative study that consider four levels: structure, rhetorical approach, linguistic level and use of information. The challenges that emerge from such written work provide valuable ideas for the development of pedagogical proposals aimed at improving the teaching of this competency.

\section{Transfer to practice}

These results seek to contribute to the line of investigation on writing in academia. Additionally, this paper is intended to serve as a basis to promote educational improvements that favour the analysis of different difficulties that university students manifest during different developmental stages of their writing competency.

\section{Palavras-chave}

Desenvolvimento de habilidades; formação profissional superior; alfabetização académica

\section{Résumé}

Cet article de recherche présente une analyse des productions écrites des étudiants universitaires récemment entrés dans un contexte d'examen. L'objectif est celui d'offrir une description détaillée de la nature linguistique-discursive de ces productions, et identifier quelques problèmes associées à la composition de ce type de textes. Pour cet objectif on a fait une étude quantitative de quatre niveaux d'analyse: la structure, la proposition rhétorique, le niveau linguistique et l'usage de l'information. Les défis qui ont émergés de dites productions donnent les indices précieux pour l'élaboration de propositions didactiques orientés à l'amélioration de l'enseignement de cette compétence.

\section{Transfert à la pratique}

Les résultats de cette étude cherchent contribuer au progrès dans la ligne de la recherche par rapport à l'écriture dans les milieux universitaires. D'une manière additionnelle, le travail qu'ici se présente a en tant qu'objectif fonctionner en tant que base pour promouvoir des meilleures didactiques qui favorisent le traitement des différents difficultés qui manifestent les étudiants universitaires dans les différents stades du développement de leur compétence écrite.

\section{Resumo}

Este artigo de pesquisa apresenta uma análise das produções escritas pelos discentes universitários recém-admitidos em um contexto de exame. O objetivo é oferecer uma descrição pormenorizada da natureza linguística-discursiva dessas produções e identificar alguns problemas associados à composição deste tipo de textos. Para tal fim foi realizado um estudo quantitativo de quatro níveis de análise: a estrutura, abordagem re tórica, nível linguístico e uso de informações Os desafios que emergem de tais produções fornecem indícios valiosos para a elaboração de propostas didáticas orientadas ao melhoramento do ensino dessa competência.

\section{Transferência à prática}

Os resultados do estudo visam contribuir ao avanço da linha de pesquisa sobre escrita nos meios universitários. Além disso, o trabalho aqui apresentado tem como objetivo server de base para promover melhoramentos didáticos a favorecer o tratamento das diferentes dificuldades manifestadas pelos discentes universitários nos diferentes estágios de desenvolvimento da sua competência escritora. 


\section{Introducción}

La atención a la escritura académica es una necesidad cada vez más sentida en las aulas universitarias de todos los ámbitos disciplinares en el contexto de la universidad española (Álvarez, 2012; Battaner, Atienza, López \& Pujol, 2001; Ezeiza, 2008; Perea, Luque \& Cepedello, 2013). Esta necesidad se manifiesta en todas las etapas formativas, tanto en el inicio de las trayectorias universitarias, como en los estudios de maestría y doctorado.

En contrapartida, se tiene una idea poco sistematizada de las necesidades de este alumnado concreto a medida que sus estudios lo van enfrentando a tener que producir discursos cada vez más especializados y que requieren un alto dominio del uso de la lengua.

El trabajo que aquí se presenta tiene, precisamente, la finalidad de obtener información detallada sobre los usos que unos estudiantes universitarios hacen del castellano en una situación de comunicación, la respuesta a preguntas de examen, para - a partir de ahí- reajustar la planificación de una práctica docente encaminada a la formación en escritura académica en los inicios de la universidad.

Así, el estudio está vinculado a una experiencia docente curricular: la asignatura Lenguaje y destrezas comunicativas I, del año académico 20102011 de la titulación de grado en educación primaria. La materia en cuestión está organizada en dos partes:

a. una primera, orientada a la mejora de las capacidades de comprensión lectora de textos académicos y

b. una segunda parte centrada en la producción escrita de tales tipos discursivos.

Precisamente, al final de la primera parte, se realizó la prueba del examen del que se recogen los datos que aquí se manejan. Una vez analizados, sus resultados se tuvieron en cuenta para perfilar la propuesta docente que se desarrollará en la segunda fase de la asignatura.

De esta manera, este trabajo se plantea los siguientes objetivos:

- Describir la naturaleza lingüístico-discursiva de las producciones escritas por estudiantes universitarios de primer curso en situación de examen.

- Poner de relieve la problemática que entraña la práctica escritora en un contexto de examen.

- Reflexionar sobre las implicaciones de los resultados obtenidos en el diseño de prácticas docentes encaminadas a la mejora del proceso de enseñanza-aprendizaje de la escritura académica de estudiantes universitarios.

Exponemos, en primer lugar, el marco teórico en el que se sustenta el trabajo (y, también, la propuesta docente vinculada a este estudio) y los diferentes aspectos de la metodología (informantes, corpus, instrumentos de recogida de datos). A continuación, nos centramos en el análisis e interpretación de los datos, para terminar con la consideración de aquellos que puedan tener implicaciones en la mejora de la propuesta docente que parte de este estudio.
Descripción del artículo | Article description | Description de l'article | Artigo descrição

Este artículo se deriva del proyecto Diagnóstico de las dificultades a las que se enfrentan los estudiantes universitarios en la producción de prácticas escritoras durante sus diferentes niveles de formación (grado, postgrado y doctorado). Estudio de caso eminentemente descriptivo de la naturaleza de la producción escrita de textos en el ámbito universitario. Se integra dentro de un macroproyecto de investigación de textos producidos por estudiantes en lengua materna (español) en diversos contextos y niveles de formación. 


\section{Las prácticas de escritura en el entorno universitario: las respuestas a preguntas de examen}

El desarrollo de este trabajo requiere que consideremos la escritura como un código lingüístico y como una práctica comunicativa que está condicionada por el contexto en el que se produce; requiere también tener en cuenta las características propias de la práctica escritora que es objeto de estudio en esta investigación, el examen.

La escritura se concibe como un código lingüístico independiente que, aunque emparentado al código oral, tiene características que le son propias (Cassany, 1989). Y como código, la escritura involucra todo un conjunto de aspectos que se sitúan en diferentes niveles del sistema de la lengua: la normativa ortográfica, las normas de puntuación, la elaboración adecuada de la frase y el párrafo y las particularidades temáticas, estructurales y de estilo de los textos.

Además, la escritura se considera como una práctica situada que varía de acuerdo con las culturas institucionales, las disciplinas y las épocas. Mijail Bajtin (1979) tuvo la importante idea de vincular texto y contexto en el estudio del uso de la lengua; esta idea fue retomada posteriormente por otros autores (Adam, 1999; Swales, 1990, entre ellos). Así, la escritura se considera como un acto social dependiente del contexto en el que se produce y de la situación retórica que lo determina (Freedman, Adam \& Smart, 1994).

También corresponde a Bajtin (1979) la consideración de las prácticas de escritura como prácticas de naturaleza dialógica que, a diferencia del discurso oral, se producen en diferido por cuanto la interacción entre emisor y receptor no tiene lugar en el mismo contexto espacio-temporal. "Todo enunciado tiene su origen en alguien y va dirigido a alguien" (Calsamiglia \& Tusón, 2008, p. 123) y, por ello, al escribir, el emisor debe tener en cuenta el acontecimiento comunicativo en el que se encuentra y a quién está dirigiendo su discurso: el destinatario.

Otro concepto esencial para comprender la naturaleza de la producción escrita como práctica comunicativa situada en un contexto histórico-social es el de comunidad discursiva (Bazerman, 1988; Maingueneau, 2004; Swales, 1990, 2004). Este concepto sitúa las prácticas escritoras como prácticas comunicativas realizadas por un grupo humano determinado para conseguir una finalidad específica y en las que autores y lectores asumen unos roles predeterminados.

En definitiva, esta idea de considerar la escritura como una práctica histórico-social supone que, al producir un texto, debemos observar y analizar el contexto en el que va a circular el mensaje. Todo ello para moldear el escrito de acuerdo a las necesidades que plantea el destinatario al que se dirige y al objetivo que se quiere alcanzar.

Vincular estas nociones con el campo de la educación plantea la necesidad de desarrollar propuestas didácticas que conduzcan a aprender los modos particulares de construir conocimiento en las diversas comunidades discursivas, y las normas, más o menos tácitas, que permiten circular y legitimar ese conocimiento generado en el seno de estas comunidades.

En este contexto resulta especialmente útil el concepto de alfabetización académica proveniente de estudios ingleses y que en el ámbito de la enseñanza del español difunde Paula Carlino (2003, 2004, 2005). Con este concepto se quiere abarcar las diversas acciones de formación desarrolladas por las universidades para "enseñar a leer y escribir" teniendo en cuenta las especificidades de las diversas áreas disciplinares. O lo que es lo 
mismo, este concepto "señala el conjunto de nociones y estrategias necesarias para participar en la actividades de producción y análisis de textos requeridas para aprender en la universidad" (Carlino, 2005, p. 13).

Una de las prácticas escritoras que más frecuentemente realizan los estudiantes universitarios, sobre todo los de los primeros cursos, para demostrar lo que han aprendido, es la respuesta a examen. Se trata de una práctica de escritura vinculada a la lectura y con la que ya están familiarizados; no es nueva, es una práctica frecuente en el quehacer escolar como instrumento de evaluación para "los estudiantes de casi todas las edades, desde escolares de 9 o 10 años, hasta los universitarios a punto de licenciarse en la enseñanza actual en España" (Battaner, Atienza, López \& Pujol, 2001, p. 7).

En este tipo de comunicación entre el profesor (quien plantea la pregunta) y el alumno (que escribe la respuesta), se espera que este último exponga conocimientos, explique ideas de los autores consultados y las aplique en el análisis de fenómenos (Grigüelo, 2004). Es decir, se espera que el alumno sea un reformulador experto, alguien capaz de exponer, de manera sintética y guardando fidelidad, lo que ha leído sobre un tema (Narvaja de Arnoux, Nogueira \& Silvestri, 2002).

Las preguntas, además de apuntar a contenidos concretos, proporcionan indicaciones sobre el "hacer" de los alumnos; les proponen acciones encaminadas hacia actividades cognitivas distintas y de diferente grado de dificultad. El alumno no solo ha de conocer los contenidos por los que se le pregunta, sino que también ha de distinguir las operaciones cognitivas y discursivas que se le proponen. Las actividades cognitivas involucradas en las preguntas de los profesores que se dan con mayor frecuencia según Liliana Grigüelo (2004) son las siguientes:

- Identificar, reconocer, distinguir, enumerar: operaciones de un grado de abstracción bajo que requieren reconocer referentes identificables.

- Definir: operación que requiere conocer el concepto para reconstruir su significado, inscribiéndolo en el contexto de la teoría a la que corresponde.

- Explicar: operación que requiere relatar la idea, problema o concepto utilizando diferentes estrategias que ayuden a su comprensión. La explicación puede tener una estructura causa-efecto o problema-solución.

- Ejemplificar: operación destinada a la ilustración de un concepto. Suele ser el complemento de una definición o una explicación. Los ejemplos de elaboración personal sirven para demostrar más la comprensión adquirida sobre el tema.

- Comparar, diferenciar: operaciones que implican considerar relaciones entre dos o más conceptos, ideas o teorías. Es necesario primero tener claro lo que se compara y luego inferir los puntos de relación y elegir la estrategia más adecuada para dar cuenta de ellos. Es importante asimismo atender a la jerarquía que presentan las distintas relaciones, ya que no todos los elementos comparados tienen el mismo grado de relevancia.

- Justificar, fundamentar, argumentar: operaciones que requieren un mayor grado de abstracción que se vinculan al discurso argumentativo. Se reconocen puntos de vista y se tienen fundamentos para justificarlos. La justificación debe ser adecuada y no debe confundirse con la opinión personal. 
En la misma línea se manifestan también Jaume Jorba, Isabel Gómez y Ángel Prat (2000) cuando proponen que desde las diferentes áreas disciplinares se enseñen a los estudiantes las habilidades cognitivo-lingüísticas necesarias para una mayor eficacia de la actividad de aprendizaje y estudio. Se trata de habilidades como describir, resumir, definir, explicar, justificar, argumentar y demostrar que los alumnos deben activar "de manera constante en la realización de las diferentes tareas que se les proponen" (Jorba, Gómez \& Prat, 2000, p. 30).

En definitiva, el alumno ha de conocer el tema por el que se le pregunta y ha de distinguir las operaciones cognitivo-lingüísticas a las que se refiere la demanda realizada por el profesor. Además, ha de ser capaz de construir un texto con el que se responda al propósito comunicativo planteado, un texto independiente, cuya lectura pueda realizarse sin tener delante la información aportada y un texto que responda a una estructura interna convencional, en el que predominan las secuencias textuales explicativas, y que es resultado de las prácticas estandarizadas para ese evento comunicativo.

\section{Metodología}

\section{Informantes y corpus}

Los informantes de esta investigación son la totalidad de los alumnos de primer curso del grado en educación primaria de la Universidad de Deusto. Se trata, por tanto de 85 alumnos, todos ellos de 18 años de edad.

El corpus, por su parte, consiste en el conjunto de textos extraídos de una de las preguntas de la prueba final que estos estudiantes realizaron para ser evaluados en la asignatura Lenguaje y destrezas comunicativas I, en el año académico 2010-2011. Esta asignatura de 6 créditos se imparte de manera obligatoria en el primer curso del grado mencionado.

Se trata, por tanto, de un corpus de textos que se han generado para un contexto real de producción, sin que los alumnos tuviesen constancia de que fuesen a ser empleados en una investigación de esta naturaleza. Son productos textuales resultado de una práctica escritora en el aula: la respuesta a una pregunta de examen, formulada de la siguiente manera:

Fíjate en los elementos paratextuales del siguiente texto y responde a la pregunta: ¿qué información obtienes de ellos y para qué te sirve esa información?

El texto al que se hace referencia es un fragmento de la obra de Carlos Lomas (1999). En este fragmento (3.5.11. Escribir), el autor cita a Jesús Tusón (1997, p. 117) para introducir su punto de vista acerca de la escritura (anexo 1).

La demanda que origina la actividad escritora responde a una de las situaciones frecuentes en los primeros cursos de formación universitaria en la medida en que se trata de evaluar lo aprendido durante el curso. Se plantea una pregunta sobre contenidos trabajados previamente: los elementos paratextuales como estrategias para intensificar la comprensión de lo que se lee. Con la actividad propuesta, se quiere valorar hasta qué punto el alumno identifica en el texto que se le proporciona los elementos paratextuales que en él aparecen (título del fragmento y lugar de la obra en el que se sitúa -3.5.11. Escribir-, referencia bibliográfica de la obra, nota a pie de página, elementos tipográficos, etc.), y si hace uso de ellos en la comprensión crítica y reflexiva del texto académico en cuestión. 
Esta actividad evaluativa es realizada por los estudiantes sin que se haya dado ningún tipo de instrucción específica o pauta acerca de cómo presentar esos conocimientos y, en consecuencia, de cómo dar respuesta a esa tarea del examen.

\section{Instrumento para la lectura de los datos}

Los instrumentos utilizados para el análisis del corpus son cuatro escalas holísticas de cinco rúbricas ${ }^{1}$, dentro de un continuum. Cada una de ellas tiene en cuenta cuatro niveles lingüístico-discursivos de los textos: Ios rasgos de la estructura del texto que se debía elaborar (anexo 2), el contexto comunicativo que se establece (anexo 3), el grado de dominio de la competencia lingüística que muestra el estudiante (anexo 4) y, finalmente, el uso que se hace de la información aprendida para resolver la demanda de la pregunta del examen (anexo 5).

Para la configuración de estas escalas se han tomado como referencia aquellas empleadas por Maite Ruiz-Flores (2009), aunque se han realizado modificaciones para que estuviesen adaptadas a las necesidades y objetivos de este trabajo.

En la lectura de los datos han participado dos expertas en el ámbito de la lengua española cuyas decisiones se han contrastado para llegar a un acuerdo en lo que respecta a la valoración de cada uno de los ítems de cada texto. Ambas investigadoras han participado en el diseño, gestión y evaluación de la asignatura por lo que estaban familiarizadas con los contenidos que se habían impartido durante el curso.

\section{Preguntas de investigación}

Con la herramienta previamente descrita se obtienen los datos que dan respuesta a las siguientes preguntas de investigación:

- Al elaborar el texto de la respuesta a examen, ¿sigue el estudiante una estructura acorde al tipo de discurso que genera?

- ¿Se emplea un registro, un tono y un léxico en relación con el contexto comunicativo en el que tiene lugar el texto que se genera?

- ¿Se manifiesta algún tipo de relación entre el dominio de la estructura propia de ese tipo de discurso y el adecuado uso del registro, el tono y el léxico?

- ¿Qué control tienen los alumnos de los aspectos formales de la construcción del texto?

- ¿Qué uso se hace de la información aprendida y/o de la que le proporciona el texto que sirve de fuente para la realización de la tarea?

\section{Resultados}

La respuesta a las preguntas planteadas se realiza a partir de un análisis cuantitativo en el que se calculan las medias, los porcentajes, las frecuencias y las correlaciones de los diferentes niveles de estudio de este trabajo. Se ofrecen los resultados en relación con la caracterización de la estructura de los textos y del contexto comunicativo que se genera, y se compara la relación entre ambos. A continuación, se exponen los resultados referentes al nivel de dominio lingüístico y al uso que se hace de la información referida para dar respuesta a la pregunta de examen.

1 La rúbrica es una guía que describe los criterios con una escala para caracterizar los niveles de ejecución con el propósito de juzgar la calidad de la tarea realizada por los estudiantes. 
Resultados referidos a la estructura y a la caracterización

del contexto comunicativo

La media (tabla 1) obtenida con respecto a la caracterización de la estructura del texto muestra un bajo control. Se aprecia una tendencia al volcado de la información que se ha aprendido, sin que haya un planteamiento del problema estructural $(37,6 \%)$-anexo 6 , informante 5 - o que trate de resolverse esta problemática mediante el uso de fórmulas polivalentes $(43,5 \%)$ :

1. "Los elementos paratextuales que podemos hallar en este texto son diferentes". Informante 3

2. "En el siguiente texto los elementos paratextuales que encontramos son los siguientes...". Informante 4

Frente a este no reconocimiento de la necesidad de plantearse una planificación de la estructura, tan solo un porcentaje acumulado de un $18,8 \%$ (correspondiente a los porcentajes de las rúbricas 3,4 y 5) resuelve deficientemente o no, aspectos estructurales en función del género textual requerido para la realización de la tarea —anexo 6 , informante 1 , como caso en el que se resuelve con éxito la estructura de acuerdo a las expectativas del tipo de discurso.

Tabla 1

Frecuencias y porcentajes en relación con la caracterización de la estructura. Los valores se exponen en el anexo 2

\begin{tabular}{cccccc}
\hline & & Frecuencia & $\begin{array}{l}\text { Porcentaje } \\
(\%)\end{array}$ & $\begin{array}{l}\text { Porcentaje } \\
\text { válido (\%) }\end{array}$ & $\begin{array}{l}\text { Porcentaje } \\
\text { acumulado } \\
(\%)\end{array}$ \\
\hline Valores & $\mathbf{1}$ & 32 & 37,6 & 37,6 & 37,6 \\
\cline { 2 - 6 } & $\mathbf{2}$ & 37 & 43,5 & 43,5 & 81,2 \\
\hline $\mathbf{3}$ & 11 & 12,9 & 12,9 & 94,1 \\
\hline $\mathbf{4}$ & 4 & 4,7 & 4,7 & 98,8 \\
\hline $\mathbf{5}$ & 1 & 1,2 & 1,2 & 100,0 \\
\hline Total & 85 & 100,0 & 100,0 & \\
\hline \hline
\end{tabular}

Fuente: elaboración propia

Con respecto a los aspectos vinculados a la caracterización del contexto comunicativo (tabla 2), en un $22,4 \%$ de los casos, los alumnos escriben sin tener en cuenta el contexto en el que se desarrolla la tarea de manera que no se presta atención ni al registro, ni al tono, ni al destinatario del texto -como ejemplo, anexo 6, informante 4-. Muy elevado es también el porcentaje de casos $(38,8)$ en los que se aprecian notas discordantes en el registro con un tono sumamente irregular -informante 3-. Estaríamos, pues, ante un porcentaje acumulado de un $61,2 \%$, muy por encima del porcentaje acumulado de estudiantes capaces de desarrollar la tarea utilizando un registro y un tono adecuado a los objetivos de la misma $(9,4 \%$, correspondiente a los porcentajes de las rúbricas 4 y 5 -ejemplo de ello se observa en el anexo 6, informante 6). 
Tabla 2

Frecuencias y porcentajes en relación con la caracterización de la situación comunicativa. Valores en el anexo 3

\begin{tabular}{cccccc}
\hline & & Frecuencia & $\begin{array}{l}\text { Porcentaje } \\
\text { (\%) }\end{array}$ & $\begin{array}{l}\text { Porcentaje } \\
\text { válido (\%) }\end{array}$ & $\begin{array}{l}\text { Porcentaje } \\
\text { acumulado } \\
\text { (\%) }\end{array}$ \\
\hline Valores & $\mathbf{1}$ & 19 & 22,4 & 22,4 & 22,4 \\
\cline { 2 - 6 } & $\mathbf{2}$ & 33 & 38,8 & 38,8 & 61,2 \\
\hline $\mathbf{3}$ & 25 & 29,4 & 29,4 & 90,6 \\
\hline $\mathbf{4}$ & 7 & 8,2 & 8,2 & 98,8 \\
\hline $\mathbf{5}$ & 1 & 1,2 & 1,2 & 100,0 \\
\hline \hline
\end{tabular}

Fuente: elaboración propia

Para conocer el grado de asociación entre las variables de estructura y planteamiento retórico se utilizó la prueba correlación de Pearson. Los análisis indican la existencia de una correlación positiva moderada entre ambas variables de $r p=, 618, p<.01$.

Tabla 3

Correlación entre los resultados de estructura y planteamiento retórico

\begin{tabular}{|c|c|c|c|}
\hline & & Estructura & Planteamiento \\
\hline \multirow[t]{3}{*}{ Estructura } & Correlación de Pearson & 1 &, $618^{* *}$ \\
\hline & Sig. (bilateral) & &, 000 \\
\hline & $\mathrm{N}$ & 85 & 85 \\
\hline \multirow[t]{3}{*}{ Planteamiento } & Correlación de Pearson & $618^{* *}$ & 1 \\
\hline & Sig. (bilateral) & ,000 & \\
\hline & $\mathrm{N}$ & 85 & 85 \\
\hline & \multicolumn{3}{|c|}{ ** La correlación es significativa al nivel 0,01 (bilateral) } \\
\hline
\end{tabular}

Sig: significatividad

N: Tamaño de la población

Fuente: elaboración propia

\section{Resultados referidos al nivel de dominio lingüistico}

Los datos analizados con respecto al dominio de la dimensión lingüística que reflejan los hablantes en esta prueba (tabla 4) evidencian un claro déficit en el conocimiento de los aspectos formales de la construcción de un texto. El análisis de aspectos como la puntuación, la ortografía, la construcción de la oración y la riqueza léxica manifiesta unos niveles de dominio deficientes. El porcentaje acumulado de los casos 1 y 2 alcanza el $68,2 \%$, lo que indica una carencia de control sobre estas dimensiones lingüísticas. Además, el número mayor de estudiantes (un total de 30) se concentra en torno a la rúbrica 1, lo que constata una ortografía en la que "abundan los errores, una puntuación deficiente con abuso de elementos paraortográficos, así como un bajo control de la estructura oracional que imposibilita la reconstrucción de las intenciones del alumno" - informante 4-. Estos casos muestran a su vez un léxico muy restringido, reiterativo e inapropiado para el contexto en el que se produce la escritura. 
El extremo opuesto queda reflejado mediante un único caso, rúbrica 5 que, acompañado de los ocho casos de la rúbrica 4, acumula un porcentaje de $10,6 \%$ alejado incluso del 21,2\% de los casos de 3. Estos últimos, con una frecuencia de 18, serían aquellos en los que "tanto la ortografía como la puntuación son aceptables, aunque con errores que no comprometen la comprensión del contenido. La sintaxis, por su parte, es básica para el desarrollo de la tarea y el léxico combina el uso de vocabulario específico con palabras excesivamente coloquiales" —informante 1-. Por ende, los textos clasificados en este grupo son textos cuya lectura exige cierta colaboración por parte del lector, ya que algunos de los errores dificultan la comprensión del significado de determinados fragmentos.

Tabla 4

Frecuencias y porcentajes en relación con el código lingüístico. Valores en el anexo 3

Frecuencia Porcentaje Porcentaje Porcentaje (\%) válido (\%) acumulado

\begin{tabular}{|c|c|c|c|c|c|}
\hline & & & & Valloo (\%) & (\%) \\
\hline \multirow[t]{6}{*}{ Valores } & 1 & 30 & 35,3 & 35,3 & 35,3 \\
\hline & 2 & 28 & 32,9 & 32,9 & 68,2 \\
\hline & 3 & 18 & 21,2 & 21,2 & 89,4 \\
\hline & 4 & 8 & 9,4 & 9,4 & 98,8 \\
\hline & 5 & 1 & 1,2 & 1,2 & 100,0 \\
\hline & Total & 85 & 100,0 & 100,0 & \\
\hline
\end{tabular}

Fuente: elaboración propia

Resultados referidos a la capacidad mostrada para referenciar las fuentes

En cuanto al uso que se realiza de la información, bien sea aprendida (tabla 5), las frecuencias permiten constatar una mayor frecuencia de resultados en el nivel 2 de la escala holística según la cual el alumno "utiliza la información para desarrollar el tema, aunque de manera parcial y deficiente". En estos resultados, el porcentaje acumulado hasta la rúbrica 2 es de $84,7 \%$, siendo la frecuencia de casos en los que no se utiliza la información para desarrollar el tema de 7, un 8,2\% - ejemplo de estas dificultades es el texto del informante 7- Del total de casos analizados, tan solo en cuatro los alumnos muestran una capacidad de utilizar correctamente la información de las fuentes para la resolución de la tarea —informante 2.

Tabla 5

Frecuencias y porcentajes en relación con el uso de las fuentes de información. Valores en el anexo 4

\begin{tabular}{cccccc}
\hline & & Frecuencia & $\begin{array}{l}\text { Porcentaje } \\
(\mathbf{\%})\end{array}$ & $\begin{array}{l}\text { Porcentaje } \\
\text { válido (\%) }\end{array}$ & $\begin{array}{l}\text { Porcentaje } \\
\text { acumulado } \\
\text { (\%) }\end{array}$ \\
\hline Valores & $\mathbf{1}$ & 7 & 8,2 & 8,2 & 8,2 \\
\cline { 2 - 6 } & $\mathbf{2}$ & 65 & 76,5 & 76,5 & 84,7 \\
\cline { 2 - 6 } & $\mathbf{3}$ & 8 & 9,4 & 9,4 & 94,1 \\
\hline $\mathbf{4}$ & 1 & 1,2 & 1,2 & 95,3 \\
\hline $\mathbf{5}$ & 4 & 4,7 & 4,7 & 100,0 \\
\hline Total & 85 & 100,0 & 100,0 & \\
\hline
\end{tabular}

Fuente: elaboración propia 


\section{Interpretación de los resultados}

Como se ha podido constatar, muy pocos estudiantes, al enfrentarse a la composición de este tipo de textos, se plantean la necesidad de dibujar un contexto comunicativo en el que se desarrolla la tarea $y$, por ende, tener conciencia de cuáles son los agentes involucrados en la situación que se genera en la composición de una respuesta a examen. En este punto, es necesario recabar más información de la que disponemos para determinar si esa falta de conciencia se debe realmente a que no disponen de las estrategias para dibujar este contexto comunicativo o si hay otro tipo de factores contextuales determinantes.

Se infiere una generalizada falta en cuanto a que un alto porcentaje de alumnos no presta atención ni al registro, ni al tono, ni al destinatario; optando en la mayoría de los casos por un discurso neutro y estándar en el que se evita cualquier tipo de relación con el receptor del producto escrito. Tan solo un alumno realiza la tarea con una conciencia explícita del lector, y de los objetivos con los que se está llevando a cabo la tarea.

Algo similar ocurre con la estructuración del texto. Los alumnos muestran una tendencia a ofrecer directamente la información que se requiere sin facilitar una introducción y a no proporcionar unas conclusiones que pongan de relieve los puntos más significativos de lo expuesto. En la mayor parte de los casos, los alumnos comienzan sus textos con la expresión de una idea que utilizan como desencadenante del desarrollo, sin que esta realmente cumpla la función de introducir el texto que elaboran.

Poner en relación ambos aspectos de la construcción de textos escritos lleva a resultados muy ilustrativos. Los datos constatan una relación directa entre el dominio de ambos: la falta de estructura va de la mano de una falta de planteamiento retórico, de la misma manera que la conciencia de necesidad de estructura también implica una conciencia de necesidad de atender al planteamiento retórico. A partir de estos resultados cuantitativos, parece necesario incidir en una aproximación más de tipo cualitativo para evidenciar hasta qué punto estas dos variables están correlacionadas en todos los estudiantes y de qué manera.

En el caso de los aspectos lingüísticos de los escritos analizados, la realidad también evidencia signos claros de bajo control de esta dimensión. El hecho de que solamente 8 de las producciones puedan valorarse como buenas o aceptables en estos términos es revelador. También lo es el que, en contra de lo que pudiera pensarse, los errores en los que incurren los estudiantes en este nivel no son solo puntuales, de superficie, ornamentales. Son errores que en el 21,2\% (rúbrica 3) de los casos dificultan la comprensión del signifi- cado de ciertos fragmentos (aunque no obstruyen la comprensión global del texto), en el 32,9\% (rúbrica 2) complican la comprensión global del texto y en el 35,3\% (rúbrica 1) se hace muy difícil la reconstrucción de las intenciones del alumno. Parece claro que, al menos en este contexto de producción de textos escritos, los estudiantes tienen muy poco control de los aspectos formales, ni siquiera de aquellos más vinculados a la representación del conocimiento alcanzado y por el que van a ser evaluados.

Del conjunto de los estudiantes que constituyen la muestra (85 en total), cuatro utilizan la información aprendida y/o la del texto del examen para resolver la tarea. Estos serían aquellos casos que apuntan los contenidos por los que se les pregunta y resuelven la operación cognitivo-discursiva que se les propone. En el caso que nos ocupa, serían operaciones de diferentes nivel de abstracción, desde identificar (lo aprendido; en este caso, "los elementos paratextuales") y definir (conocer los conceptos y demostrar que se han aprendido), hasta explicar y ejemplificar (tener en cuenta el grado de pertinencia respecto del tema planteado) (Grigüelo, 2004; Jorba, Gómez \& Prat, 2000).

En este marco, resulta reseñable el rendimiento académico escaso que evidencian los textos de los estudiantes restantes. Para ellos, la información que pueden inferir del texto que se proporciona para realizar la tarea no pasa de ser un conocimiento inerte que no pueden aplicar para resolver la tarea planteada en toda su complejidad (Whitehead, 1929). Es un conocimiento puramente enunciativo que se obtiene con una recepción pasiva y que no es fruto de procesos genuinos de comprensión. David P. Ausubel (2002) hablaba en términos de aprendizaje memorístico y aprendizaje significativo que opondría los enunciados memorizados versus una asimilación y anclaje de los conocimientos en la estructura cognitiva del aprendiente.

Podemos decir que los resultados apuntan a lo indicado por Maite Ruiz-Flores (2009) cuando habla de que el alumno genera una serie de pseudotextos en los que, además de no intervenir todos los procesos cognitivos implicados en la escritura, se "dice el conocimiento" - en términos de Marlene Scardamalia y Carl Bereiter (1992) - sin que tenga lugar la construcción de un nuevo conocimiento y sin que tenga lugar, por ende, la función epistémica de la escritura (Castelló, 2006; Wells, 1987).

En Argentina, el proyecto de investigación llevado a cabo recientemente por el Instituto Provincial de Educación Superior de Santa Cruz (Vázquez, Serón, Melani, Berrocal, Dávila \& Felmer, 2009) confirma los resultados de este trabajo, pues en él también se ponen en evidencia las escasas marcas de construcción de un destinatario textual que permitirían inferir que el texto está producido para un lector determinado, 
en este caso, el docente. Con respecto al nivel textual, "en muy pocas respuestas los conceptos se explicitan organizados por párrafos y relacionados lógicamente" (Vázquez, Serón, Melani, Berrocal, Dávila \& Felmer, 2009, p. 14) y no hay un uso del léxico como herramienta conceptual explicativa. Por último, en cuanto al dominio del código, los informantes de este proyecto reflejan, al igual que ocurría en nuestro caso, falta de dominio de los recursos ortográficos y/o morfosintácticos de la lengua.

\section{Implicaciones didácticas}

Estos resultados no hacen más que evidenciar la necesidad de incidir en la didáctica de la escritura en el ámbito universitario desde niveles iniciales. Pero, ¿cómo llevar a cabo esta formación? Parece indispensable que los docentes cuenten con una serie de pautas o mecanismos de instrucción que les ayuden a realizar el acompañamiento y guía que los estudiantes necesitan para ir entrando en la nueva comunidad discursiva en la que se están formando, y esto con todas las prácticas escritoras que vayan teniendo que ser capaces de producir. Incluso con aquellas que forman parte de su quehacer en el aula mucho antes del comienzo de sus estudios en la universidad como es el caso de la respuesta a una pregunta de examen.

Es importante que el dispositivo de instrucción que se diseñe se plantee de manera que el estudiante sienta la necesidad de esta formación. Asimismo, esta tendrá que pasar a formar parte de los criterios de evaluación empleados por el docente. A partir de aquí, cabe insistir, a tenor de los resultados obtenidos en este trabajo, en la conveniencia de atender a los siguientes aspectos:

a. Cuestiones locales relativas al componente lingüístico

Lejos de ser consideradas cuestiones locales solo de tipo formal, dentro del componente lingüístico es necesario atender sobre todo aquellos aspectos que dificultan la reconstrucción de las intenciones del mensaje. Entre otros, estos problemas pueden venir determinados por la alteración del orden lógico de la oración, la inconsistencia en la cohesión de los componentes del párrafo e, incluso, de la oración, errores ortográficos de carácter diacrítico, el uso inapropiado de los signos de puntuación, y otros.

b. Cuestiones globales como las derivadas de la situación comunicativa en la que se produce el texto

Durante la fase de planificación del texto, es necesario que el alumno establezca un marco de actuación que tenga en cuenta no solo el tema sobre el que va a hablar, sino también el propósito de esa comunicación, a quién se está dirigiendo y otras posibles restricciones que deriven de la situación comunicativa. Asimismo, este marco de actuación afectará la textualización y el modo en que se afrontan la revisión y corrección del texto.

c. Cuestiones relativas al tipo de práctica escritora a la que se enfrenta en cada momento

En el caso de la respuesta a una pregunta de examen, parece clara la necesidad de hacer consciente al estudiante de que este contexto particular de producción escrita exige no solo tener en cuenta el contenido informativo sobre el que se pregunta, sino también los requerimientos cognitivo-lingüísticos que entraña. Se trata, por tanto, de que el alumno vaya más allá de exponer un conocimiento declarativo y sea capaz de hacer un uso adecuado del saber del que dispone. 
Al hilo de estas sugerencias, cabría recuperar la idea que presenta Paula Carlino (2005) de que la responsabilidad de alfabetizar académicamente al alumno recae en todos los integrantes de la comunidad académica y no solo en aquellos profesores que tienen como objetivo final de la asignatura el desarrollo de la competencia comunicativa escrita. Se trata de una responsabilidad compartida que tendría como fin erradicar las contradicciones que presenta un sistema educativo que, en muchos casos, no se plantea la necesidad de "enseñar a escribir", pero que utiliza esta práctica como vía para evaluar a los alumnos.

Así, además de despertar la conciencia de los agentes implicados en este proceso de enseñanza-aprendizaje, sería conveniente diseñar propuestas didácticas en las que la escritura sirva como herramienta para aprender una materia de manera acorde a los géneros propios de un campo disciplinar (Carlino, 2006; Bazerman, Little, Chavkin, Fouquette, Bethel \& Garufis, 2005).

\section{Conclusiones}

En este trabajo, hemos identificado las dificultades que afrontan los estudiantes recién iniciados en la universidad a la hora de enfrentarse a responder una pregunta en situación de examen. Como se ha podido ver, los instrumentos utilizados para la lectura de los datos permiten obtener una fotografía de los desafíos y las dificultades a los que hacen frente los estudiantes.

La actividad escritora que realizan está marcada, sobre todo y, en general, por atender a la enunciación de los conceptos o ideas presentes en la pregunta hecha por el profesor. En pocos casos, esta actividad está condicionada por las operaciones cognitivo-lingüísticas que exige la tarea que el profesor plantea en su pregunta; y, por la necesaria interacción que el estudiante ha de establecer con su interlocutor que es, en este caso, quien va a evaluar lo que ha aprendido.

En consecuencia, el resultado de esta actividad evidencia desafíos importantes, estrechamente vinculados: un aprendizaje que el alumno no hace suyo y le cuesta utilizarlo en el análisis de situaciones nuevas; y unos escritos que reflejan poco control de los aspectos formales de uso de la lengua, incluso de aquellos que comprometen la representación del conocimiento por el que van a ser evaluados.

Las implicaciones que esta fotografía tiene en los procesos de enseñanzaaprendizaje de la competencia escritora del ámbito académico parecen claras y quedan reafirmadas por trabajos realizados en esta misma línea sobre diferentes géneros de este mismo ámbito (Narvaja de Arnoux, Nogueira \& Silvestri, 2002, 2006; Narvaja de Arnoux, 2009; entre otros). Este estudio, además, ofrece una serie de pautas didácticas que, adaptadas a las diversas situaciones del aula, permiten al profesor intervenir para la mejora de la formación en esta competencia de manera transversal en el plano curricular de cualquier titulación de grado.

Antes de finalizar, es importante mencionar las limitaciones de este estudio. Es evidente que, sin realizar investigaciones más exhaustivas y que incluyan otras prácticas escritoras de los comienzos de los estudios universitarios, no pueden extrapolarse los resultados a la realidad del conjunto de esta comunidad. Del mismo modo, cabe considerarlos, fundamentalmente, como un primer acercamiento a la realidad de unas producciones que forman parte del ámbito universitario, pero cuya enseñanza no ha sido sistematizada. Los hallazgos de este trabajo son, por tanto, una "fotografía", un estudio exploratorio cuyo fin es poner de relieve los desafíos que enfrentan los estudiantes para establecer un marco sobre el cual realizar investigaciones enfocadas en diferentes aspectos.

Entre las futuras pesquisas, cabría llevar a cabo investigaciones experimentales en las que se indague sobre los diversos aspectos que han servido para caracterizar la producción de los informantes de este estudio, a saber: la 
estructura, la génesis de un contexto comunicativo, el dominio del nivel lingüístico y el uso de la información. Así también, llevar a cabo proyectos de mejora de la formación de la competencia escrita de estos alumnos independientemente del campo de estudio en el que se encuentren y en los que los profesores sean parte activa de los mismos. Como segunda parte de esta investigación ya iniciada, se sugiere analizar de manera cualitativa los textos con el fin de conocer aspectos intrínsecos a la producción de los informantes en este contexto de producción.

\section{Sobre las autoras}

Manuela Álvarez-Álvarez es profesora titular en la Universidad de Deusto y miembro del equipo de investigación Desarrollo de competencias y valores. Labor docente e investigadora en el campo de la metodología de enseñanza de lenguas y en la formación en escritura académica.

María del Mar Boillos-Pereira es investigadora predoctoral de la Universidad de Deusto y becaria del equipo de investigación Desarrollo de competencias y valores. Investigación en el campo de la adquisición del español por arabófonos y en la escritura en lengua materna y extranjera.

\section{Referencias}

Adam, J.-M. (1999). Linguistique textuelle. Des genres de discours aux textes. Paris: Nathan/HER.

Álvarez, M. (2012). La relevancia de las competencias comunicativas en la formación del estudiante universitario y retos que plantea. En Mercedes Acillona-López (ed.). Marcos interpretativos de la realidad social contemporánea, 29-41. Bilbao: Publicaciones de la Universidad de Deusto.

Ausubel, D. (2002). Adquisición y retención del conocimiento: una perspectiva cognitiva. Barcelona: Paidós.

Bajtin, M. (1979). El problema de los géneros discursivos. En Estética de la creación verbal, 248-293. México: Siglo XXI. Disponible en: https://circulosemiotico.files.wordpress.com/2012/10/estetica-de-lacreacic3b3n-verbal.pdf

Battaner, P.; Atienza, E.; López, C. \& Pujol, M. (2001). Aprender y enseñar: la redacción de exámenes. Madrid: Antonio Machado Libros.

Bazerman, C. (1988). Shaping Written Knowledge. The Genre and Activity of the Experimental Article in Science. Madison: University of Wisconsin Press. Disponible en: http://wac.colostate.edu/books/bazerman_ shaping/shaping.pdf

Bazerman, C.; Little, J.; Chavkin, T.; Fouquette, D.; Bethel, L. \& Garufis, J. (2005). Writing across the Curriculum. Reference Guides to Rhetoric and Composition. West Lafayette, Indiana: Parlor Press LLC. Disponible en: http://wac.colostate.edu/books/bazerman_wac/wac.pdf

Calsamiglia, H. \& Tusón, A. (2008). Las cosas del decir. Barcelona: Ariel.

Carlino, P. (2003). Alfabetización académica: un cambio necesario, algunas alternativas posibles. Educere. Revista Venezolana de Educación, 6 (20), 409-420. Disponible en: http://www.redalyc.org/pdf/ 356/35662008.pdf

Carlino, P. (2004). Escribir a través del currículum. Tres modelos para hacerlo en la universidad. Lectura y Vida, Revista Latinoamericana de Lectura, 25 (1), 16-27. Disponible en: http://media.utp.edu.co/referencias-bibliograficas/uploads/referencias/articulo/248-escribir-a- 
travs-del-curriculum-tres-modelos-para-hacerlo-en-la-universidadpdf-jQyDB-articulo.pdf

Carlino, P. (2005). Escribir, leer y aprender en la universidad: una introducción a la alfabetización académica. Buenos Aires: Fondo de Cultura Económica, FCE.

Carlino, P. (2006). Algunas propuestas probadas en talleres de escritura de tesis. Coloquio internacional de Escritura y producción de conocimiento en carreras de posgrado. Cátedra UNESCO de lectura y escritura. Buenos Aires: UNESCO. Disponible en: http://www.aacademica.com/paula. carlino/19

Cassany, D. (1989). Describir el escribir: cómo se aprende a escribir. Barcelona: Paidós.

Castelló, M. (2006). La escritura epistémica: enseñar a gestionar y regular el proceso de composición escrita. I Congreso Internacional de Investigación, Educación y Formación Docente. Universidad de Antioquia, Medellín, Colombia, 30, 31 de agosto y 1 de septiembre.

Ezeiza, J. (2008). Las competencias comunicativolingüísticas de tipo disciplinar: perfiles profesionales y recursos para su desarrollo en el nuevo EEES [Espacio Europeo de Educación Superior]. Actas del XXXVII Simposio Internacional de la Sociedad Española de Lingüística, 131-144. Disponible en: http://www.ehu.eus/PAT/compe/ lanak/Ezeiza_2.pdf

Freedman, A.; Adam, C. \& Smart, G. (1994). Wearing Suits to Class: Simulating Genres and Simulations as Genre. Written Communication, 11 (2), 193-226.

Grigüelo, L. (2004). El parcial universitario. En Sylvia Nogueira. Manual de lectura y escritura universitarias. Buenos Aires: Biblos.

Jorba, J.; Gómez, I. \& Prat, Á. (2000). Hablar y escribir para aprender. Uso de la lengua en situación de enseñanza-aprendizaje desde las áreas curriculares. Madrid: Síntesis.

Lomas, C. (1999). Cómo enseñar a hacer cosas con palabras. Teoría y práctica de la educación lingüística, vol. I. Madrid: Paidós.

Maingueneau, D. (2004). Retour sur une categorie: le genre. En Jean-Michel Adam, Jean-Blaise Grize, Magid Ali-Bouacha (eds.). Texte et discours: catégories pour l'analyse, 107-118. Dijon: Université de Dijon.

Narvaja de Arnoux, E. (dir.) (2009). Escritura y producción de conocimiento en las carreras de posgrado. Buenos Aires: Santiago Arcos.

Narvaja de Arnoux, E.; Nogueira, S. \& Silvestri, A. (2002). La construcción de representaciones enunciativas: el reconocimiento de voces en la comprensión de textos polifónicos. Revista Signos: Estudios de Lingüística, 35 (51-52), 129-148. Disponible en: http://www.scielo.cl/scielo.php?script=sci_arttex t\&pid $=$ S0718-09342002005100010

Narvaja de Arnoux, E.; Nogueira, S. \& Silvestri, A. (2006). Comprensión macroestructural y reformulación resuntiva de textos teóricos en estudiantes de institutos de formación de docentes primarios. Revista Signos: Estudios de Lingüística, 39 (60), 9-30. Disponible en: http://www. scielo.cl/scielo.php?script=sci_arttext\&pid= S0718-09342006000100001\&lng=es\&nrm= iso\&tlng $=$ es

Perea, F.; Luque, L. \& Cepedello, M. (2013). Metodología y evaluación de la actividad escrita. En Francisco Javier Perea-Siller (coord.). Comunicar en la universidad. Descripción y metodología de los géneros académicos, 51-74. Córdoba: Servicio de publicaciones de la Universidad de Córdoba.

Ruiz, M. (2009). Evaluación de la lengua escrita y dependencia de lo literal. Barcelona: Graò.

Scardamalia, M. \& Bereiter, C. (1992). Dos modelos explicativos de los procesos de composición escrita. Infancia y Aprendizaje, 58, 43-64. Disponible en: dialnet.unirioja.es/descarga/articulo/48395.pdf

Swales, J. (1990). Genre Analysis: English in Academic and Research Settings. Cambridge: Cambridge University Press.

Swales, J. (2004). Research Genres. Explorations and Applications. Cambridge: Cambridge University Press.

Tusón, J. (1997). La escritura. Una introducción a la cultura alfabética. Barcelona: Octaedro.

Vázquez, L. (dir.); Serón, M. I.; Melani, S.; Berrocal, V.; Dávila, E. \& Felmer, E. (2009). Los procesos de producción y comprensión de la lengua escrita en alumnos del ISFD [Instituto Superior de Formación Docente] y las características que asume la evaluación de las producciones de los estudiantes. Santa Cruz: Instituto Provincial de Educación Superior, IPES, Caleta Olivia. Disponible en: http://ipesco.scr.infd.edu.ar/sitio/ upload/169.pdf

Wells, G. (1987). Aprendices en el dominio de la lengua escrita. En Amelia Álvarez (comp.). Psicología y Educación: realizaciones y tendencias actuales en la investigación y en la práctica: actas de las II Jornadas Internacionales de Psicología y Educación, 57-72. Madrid: Ministerio de Educación y Ciencia.

Whitehead, A. N. (1929). The Aims of Education and Other Essays. New York: MacMillan. 


\section{Anexos}

Anexo 1

Examen

\subsubsection{Escribir}

Lee con atención el siguiente texto de Jesús Tusón:

Todo el que escribe ha recibido la herencia de una larga tradición iniciada hace más de cinco mil años en tierras de Mesopotamia, y también depende de toda la tecnología que empezó a desarrollarse en Maguncia, hace ahora más de medio milenio, y con la cual se desplegó la producción mecánica del libro. Umberto Eco ha dicho, más de una vez, que "no hay libro sin libros", y eso quiere decir, más en general, que cualquier escrito tiene como trasfondo otros escritos, y todos se amoldan a unas reglas (de ortografía, de puntuación y algunas más que veremos) que los hacen posibles o, al menos, aptos para la lectura. Ahora bien la existencia de letra y de las normas de escritura no garantizan la bondad comunicativa (y menos aún la estética) del producto final: un instrumento tan poderoso como la escritura puede ser utilizado con impericia, descuido y de forma torpe, y el resultado de todo ello será un texto ininteligible o desorganizado... aunque quizá perfecto desde el punto de vista de la ortografía y de la puntuación. La conquista, pues, de la escritura habría de ser correlativa de un uso digno, transparente, desde la perspectiva comunicativa y explorador de todas sus posibilidades: las de orden expositivo, las de precisión conceptual y las de expresividad y estilo. A menudo, un escritor o una escritora son sometidos a la pregunta de si "nacen" o si "se hacen" en el campo de su profesión, y a veces la respuesta a esta pregunta trivial [...] deja a los lectores de la entrevista (quizá aspirantes a escritores) bastante desanimados: hay una parte — dicen - que pertenece al genio natural, y eso se tiene o no se tiene, y otra parte que puede llegar a conseguirse con la práctica de la lectura y de la escritura. Pero si prescindimos de misticismos, habilidades orales [...], y que la maestría de la escritura no es más que una técnica que todo el mundo puede llegar a dominar en niveles diversos [...]. Cualquier otra posición referida al "genio" con que algunos llegan al mundo no es sino la expresión ególatra de un aislamiento que nadie quiere confesar con palabras directas.

Un buen planteamiento inicial para el control del texto escrito es saber situarse, a conciencia, dentro del esquema general de la comunicación, conocido por todos y muy sencillo, que aquí adaptamos al acto de escritura: un emisor (una persona) escribe un mensaje (un texto) dirigido a un receptor (otra persona, o muchas). Este mensaje o texto habla de algo (el referente o tema) y está escrito en una lengua cuyo conocimiento es compartido por el emisor y por los receptores (esta lengua es el código común). Finalmente, el texto tiene un soporte (llamado canal de transmisión) con el que se materializa el acto de la comunicación escrita.

De todo este conjunto de factores, vale la pena tener muy en cuenta, en primer lugar, el punto de recepción del escrito. Dicho de otra forma, es necesario que quien escriba tenga "piedad" de los lectores, que sepan que se encontrarán solos ante el texto y que, en la inmensa mayoría de los casos, el diálogo aclaratorio con la fuente productora del mensaje será absolutamente imposible. La soledad de la lectura, pues, requiere textos claros, ordenados y autosuficientes. Y todo ello pasa, previamente, por el trabajo paciente de la elaboración textual, muy alejada de la espontaneidad que algunos predican a veces.

(Jesús Tusón, La escritura. Una introducción a la cultura alfabética, Barcelona, Octaedro, 1997, págs. 117-118)

Carlos Lomas. Teoría y práctica de la educación lingüística. Cómo enseñar a hacer cosas con palabras. Barcelona, Paidós, 1999, vol. I, págs. 356-357.

Recorte del examen proporcionado a los estudiantes 
Anexo 2

Escala holística sobre la estructura del texto

1. Texto como mera transcripción gráfica del discurso oral sin control de la estructura (carece de párrafos o hay una distribución arbitraria de estos). / No reconoce la necesidad de plantearse problemas estructurales, ni crear la sensación de introducción o final de texto.

2. Presenta un bajo control de la estructura del texto que está dominada por el volcado de ideas o de información de la fuente. / No plantea problemas estructurales o trata de resolverlos mediante la aplicación de fórmulas polivalentes.

3. El género textual es reconocible y se responde convencionalmente a sus requerimientos, se resuelven deficientemente algunos aspectos estructurales o recurre a la aplicación de convenciones extrapolables y consabidas. / Relación aceptable entre el uso de esquemas textuales y objetivos de contenido.

4. Uso de estructuras reconocibles y escribe dentro de las convenciones del género textual adecuado a la tarea.

5. La selección de organizadores textuales y la distribución en párrafos están al servicio de objetivos concretos. Muestra un uso consciente de la estructura. / Resuelve adecuadamente los problemas estructurales planteados.

Anexo 3

Escala holística de la caracterización del contexto comunicativo

1. El registro utilizado presenta fuertes notas discordantes con lo convencionalmente esperado. / Escribe sin representarse a un lector-destinatario de esa tarea de redacción concreta, o como si escribiera un texto privado que no fuera para nadie. / No utiliza conscientemente una voz para desarrollar la tarea. / Ausencia de algo que pueda llamarse tono del escrito, debido a que las decisiones sintácticas y léxicas no están al servicio de los objetivos.

2. Registro con notas discordantes con lo convencionalmente esperado. / El tono del escrito resulta sumamente irregular y no parece producto de decisiones conscientes.

3. El registro utilizado cae en general dentro de lo convencionalmente esperado, aunque haya ciertos usos fuera del espectro de expectativas proyectado por la tarea. / Presenta control moderado del tono del escrito, aunque no siempre es homogéneo ni se adecúa a los propósitos de la tarea.

4. El registro léxico se adecúa a los objetivos de la tarea. / El tono del escrito se corresponde con lo esperado para el género textual,

5. Dominio del registro léxico y del tono, en función de los objetivos de la tarea, así como una conciencia explícita de su lector.

Anexo 4

Escala holística de la dimensión lingüística

1. Ortografía con muchos errores. / Puntuación muy deficiente (párrafos amalgamados o sin estructura y uso de puntuación paraortográfica). / Bajo control de la estructura oracional, con errores que pueden afectar a todos los niveles. / Léxico restringido o inapropiado, con numerosas repeticiones. / La lectura del texto es dificultosa y los numerosos errores imposibilitan la reconstrucción de las intenciones del alumno.

2. Ortografía con bastantes errores. / Puntuación con errores (falta puntuación, comas incorrectas, no se introduce adecuadamente el estilo directo, etc.). / Bajo control de la estructura oracional. Errores en el nivel sintáctico e inferiores (anacolutos). / Léxico restringido o inapropiado. (Falta de dominio de recursos de correferencia). / El texto no se lee de manera fluida y los numerosos errores dificultan la comprensión general del texto.

3. Ortografía aceptable con algunos errores. / Puntuación aceptable con errores que no comprometen la comprensión del contenido. / Sintaxis oracional demasiado básica para desarrollar la tarea. (Algún error de sintagma). / El léxico combina el uso de vocabulario específico con palabras excesivamente coloquiales para desarrollar la tarea. / La lectura del texto requiere cierta colaboración por parte del lector; algunos errores dificultan la comprensión del significado en ciertos fragmentos de texto pero no obstruyen su comprensión global.

4. Ortografía correcta, aunque puede haber algún error. / Puntuación correcta aunque no se haga uso de todos los recursos. / Sintaxis oracional correcta en general, aunque puede haber poca variación. / Selección léxica adecuada, pero pueden presentarse repeticiones excesivas. / El texto se lee con facilidad, sin que errores ni puntuación obstruyan la comprensión del contenido.

5. Ortografía correcta. / Puntuación correcta y adecuada a los propósitos de la tarea. / Sintaxis oracional correcta y variada. / Selección léxica adecuada a los propósitos de la tarea. / El texto se lee de manera fluida y la transición de párrafos está bien lograda. 
Escala holística del uso de la información

1. No utiliza los contenidos trabajados para desarrollar el tema.

2. Utiliza los contenidos trabajos para desarrollar el tema, aunque de manera parcial y deficiente.

3. Uso parcial pero correcto de los contenidos trabajados.

4. Utiliza toda la información pero no es capaz de interpretarla en función de las consignas de la tarea.

5. Utiliza correctamente la información de las fuentes propuestas para la tarea.

Anexo 6

Ejemplos de fragmentos de las producciones de 9 informantes. Transcripciones fieles

\section{Informante 1}

Los elementos paratextuales son aquellos elementos que sin ser parte vital del texto nos da información sobre este. Algunos son más importantes que otros ya que la información que contienen revelan mucha información sin la cual el texto no sería tan fácil de comprender. He aquí la lista de los elementos paratextuales de este fragmento:

*3.5.11. Escribir $\rightarrow$ Esto nos indica que el fragmento que hay a continuación es parte de otro texto mayor y nos indica el posible tema a tratar, escribir.

*[...] $\rightarrow$ este elemento nos indica que el fragmento ha sido acortado, pero no de cualquier manera ya que el elemento está colocado en el preciso lugar de dónde se suprimió el texto. Puede ser porque la información no era relevante o tal vez porque era muy confusa para los lectores a los que estaba adaptado.

$*(x) \rightarrow$ el texto que va entre paréntesis nos da información adicional, de manera que la comprensión de la idea planteada anteriormente sea más completa y correcta. Los ejemplos y/o aclaraciones siempre son beneficiosos.

*Al final del fragmento nos encontramos también con información adicional la cual nos indica quién era el escritor, a qué parte del libro pertenece el fragmento, a qué libro pertenece, dónde fue editado el libro (lugar geográfico y editorial) y año de publicación, así como las páginas del libro en las que encontraremos dicho fragmento. **

En resumen, toda esta información obtenida me sirve para tener información adicional que no obtendría leyendo simplemente el texto. Eso me ayuda a comprender mejor el contexto y por tanto las ideas que se presentan.

**Además, también aparece esa misma información si este fragmento ha sido adaptado o introducido en otro libro. En este último deberán aparecer los detalles del primero.

\section{Informante 2}

Para empezar, nos aparece "3.5.11. Escribir", lo cual nos indica que el siguiente texto que vamos a leer pertenece a uno de los apartados del libro y que en el índice queda jerarquizado de esa manera. Nos podemos hacer una idea del libro en cuanto a su estructuración, lo cual nos ayudará a situarnos dentro del libro.

Por otra parte, aparece el siguiente mandato:

"Lee con atención el siguiente texto de Jesús Tusón": 
Esto nos indica que este fragmento va dedicado a una de las instrucciones que el autor ha hecho. De esta manera podemos imaginarnos que el libro tiene un objetivo didáctico.

Además aparecen varios puntos suspensivos entre corchetes que nos señalan que en los lugares que se encuentran había más texto.

Por último, nos encontramos con dos bibliografías. Primero está la que nos indica dónde podemos encontrar el texto que vamos a leer. La segunda pertenece al autor del libro en el que nos hemos encontrado el fragmento. Las dos bibliografías nos pueden servir para acudir a los libros pertenecientes de ese apartado y poder ampliar nuestro conocimiento sobre el tema que trata el texto.

\section{Informante 3}

Los elementos paratextuales que podemos hallar en este texto son diferentes.

En primer lugar podemos leer el título del texto. El título lo encontramos dentro del elemento paratextual denominado: portada.

[En segundo lugar encontramos el elemento paratextual Ilamano: bibliografia] tachado

Este elemento paratextual nos sirve para tener un previo conocimiento de lo que va a tratar el texto, que en este caso tratará sobre la escritura.

En segundo lugar, encontramos el elemento paratextual llamado: bibliografía.

La bibliografía nos informa sobre la cita que utiliza del hombre llamado: Jesús Tusón. Al mismo tiempo también nos informa del año y el lugar en el que fue publicado este libro: es decir en Barcelona, en el año 1999.

\section{Informante 4}

En el siguiente texto los elementos paratextuales que encontramos son los siguientes: bibliografias, las cuales aparecen al final del texto. Por un lado, aparece la indicación de a quién pertenece el fragmento que acabamos de leer: (Jesús Tusón, La escritura. Una introducción a la cultura alfabética, Barcelona, Octaedro, 1997, págs. 117-118). Además de esta referencia también aparece la del libro, es decir: (Carlos Lomas, Teoría y práctica de la educación lingüística. Cómo enseñar a hacer cosas con palabras. Barcelona, Paidós, 1999, vol. I, págs. 356-357).

Con estas referencias bibliográficas sabemos por una parte quien es el que a escrito el fragmento que hemos leido, es decir, Jesús Tusón, de que libro esta sacado, cuando se escribio, donde y las páginas en donde aparece este fragmento en ese libro. Pero es que además, este fragmento esta en otro libro, el cual esta escrito por Carlos Lomas, y también sabemos con la segunda referencia bibliográfica, cómo se llama el libro, cuando se escribio, donde y en que páginas esta el fragmento que hemos leido.

Con esta información podemos saber un poco sobre que va tratar ese apartado, conociendo un poco al autor y con el nombre del título, ya que nos puede dar una previa información. Por eso, leyendo y teniendo en cuenta estos elementos paratextuales, podríamos saber un poco sobre que puede ir el libro, ya que podríamos llegar a deducirlo por el título o con el autor.

\section{Informante 5}

Los elementos paratextuales, tienen la finalidad de darnos una información previa antes de leer el texto.

En este texto, gracias a los elementos paratextualse, podemos observar quienes son los autores del siguiente texto, en este caso, Jesús Tusón, Carlos Lomas. En que páginas del libro se encuentra el fragmento (117-118), (356-357), el año en que está escrito, el título. 


\section{Informante 6}

Elementos paratextuales

En el texto que tenemos delante aparecen varios elementos paratextuales, que nos ayudan a tener información suplementaria sobre el texto que vamos a proceder a analizar. Estos elementos son frases o palabras que sin pertenecer al completo texto hacen referencia a este y le dan cierta explicación.

Para comenzar nos fijamos en las palabras que aparecen antes de incluso comenzar el texto: "3.5.11. Escribir" y "lee con atención el siguiente texto de Jesús Tusón". Estas dos frases son elementos paratextuales que nos ayudan a ver que el texto que tenemos delante pertenece a una obra, la cual tiene subapartados y estos a su vez otros. De ahí que aparezcan los números 3.5.11. Además, nos damos cuenta de que en el apartado al que pertenece este fragmento se trata de la escritura. Por otra parte, con la frase "lee con atención el siguiente texto de Jesús Tusón", deducimos que el autor de este fragmento ha decidido introducir por la razón que sea un texto de otro autor, en este caso, de Jesús Tusón. Es decir, con este último elemento paratextual sacamos en conclusión que el autor del apartado 3.5.11. Escribir no es el autor del texto que tenemos delante.

Otros elementos paratextuales que aparecen en el texto son las dos diferentes referencias que están colocadas al final del fragmento seleccionado. En ambas referencias, la primera del texto de Jesús Tusón y la segunda del texto de Carlos Lomas, aparecen elementos similares. Primero aparece el autor del fragmento de Carlos Lomas ha seleccionado, Jesús Tusón. Después, el título de la obra de la que se ha extraído dicho fragmento, "La escritura. Una introducción a la cultura alfabética". Más tarde, aparece la editorial en la que ha sido publicada la obra y el año, BarceIona: Octaedro, 1997. Para finalizar aparecen las páginas exactas de las que se ha sacado este texto, págs. 117-118. En la segunda referencia pasa exactamente lo mismo. Aparece el autor, Carlos Lomas; el título de la obra, "Teoría y práctica de la educación lingüística. Cómo enseñar a hacer cosas con palabras"; la editorial, Barcelona: Paidós; el año de la publicación, 1999; el primer volumen, y las páginas de las que se ha extraído este texto, págs. 356-357.

\section{Informante 7}

Gracias a los elementos paratextuales de este texto como [...], etc. he llegado a la comprensión total de este texto.

La información que obtengo de ellos es que una escritura buena y adecuada consiste, por parte de unos, en una habilidad que tiene uno dentro, y por otra parte, se puede llegar a conseguir con la práctica.

Una buena estructura es vital para la comprensión de un texto, empezando por un emisor, acabando en el soporte (canal de transmisión). Un buen escritor debe escribir de una manera muy clara, y los textos deben ser ordenados y autosuficientes.

En cuanto al autor del texto, es claramente Jesús Tusón, y lo escribió en 1997. 